\title{
Supporting Alzheimer's residential care A novel indoor localization system
}

\author{
Andrea Masciadri, Ilaria Carlini, Sara Comai and Fabio Salice \\ Department of Electronics, Information and Bioengineering Politecnico di Milano \\ via Anzani 42, 22100, Como, ITALY \\ \{andrea.masciadri,sara.comai,fabio.salice\}@polimi.it, ilaria.carlini@mail.polimi.it
}

\begin{abstract}
Keywords: Indoor Localization System, Android Mobile Application, iBeacon, Assistive Technology, Internet Of Things
Abstract: This work illustrates a localization system specifically designed to be applied in "Il Paese Ritrovato", a highly innovative health-care facility for people affected by Alzheimer's disease in Monza, Italy. Patients are provided with an iBeacon bracelet broadcasting data packets that are collected through the use of a dense network of devices acting as receiving antennas. The system evaluates the path-loss of the received signal and corrects the computed position with a probabilistic approach to avoid wall-crossing. Localization data are merged with information from other IoT devices such as smart sensors, appliances and expert annotations; the resulting dataset will be extremely important to analyze behaviors, habits and social interactions among patients.
\end{abstract}

\section{INTRODUCTION}

The growing longevity and declined fertility rate of the industrialized countries have been shifting the age distribution of population towards increasingly elder ages. This shift will continue to dangerously accelerate and, according to the United Nation forecast (United Nations and Affairs, 2017), in 2030 the number of people older than 60 years old will grow to 1.4 billions worldwide. The projection for 2050 shows an increment to more than double of the 2017 size of the selected age group, reaching an increment of $116 \%$, surpassing the number of adolescent and youth aged.

A factor that has been proven to be largely correlated to aging is the manifestation of dementia. Approximatively $3 \%$ percent of people between the ages of 65 and 74, 19\% between 75 and 84, and nearly half of those over 85 years of age have dementia (Umphred, 2012). Dementia is a syndrome characterized by symptoms related to memory, problemsolving, language impairments and other compromised cognitive skills that affects the regular and everyday activities of a person.

Alzheimer's disease is the most common form of progressively disabling degenerative dementia with onset predominantly in presenile age. It is estimated that about $60-70 \%$ of dementia cases are due to this condition, while $10-20 \%$ to vascular dementia (World Health Organization, 2015). Numerous studies have been conducted to identify a possible treatment for
Alzheimer's disease, but currently no definitive therapies has been found and due to the progressive course of the disease, the management of patient's needs becomes essential. The ultimate goal of the care provided by caregivers is to extend as much as possible the maintenance of cognitive abilities, to decelerate the progression of neuronal degeneration and, through a series of specific daily activities, to encourage the development of a natural brain compensation. Keeping the patients active with daily activities is therefore a prerequisite of the long term treatment centers. In order to create a stimulating environment, that is also able to accommodate people and make them feeling at home, it is required to design a new type of health care infrastructure.

The principal known methodologies used to estimate a human position in an indoor localization system can be divided into three categories (Farid et al., 2013; Tariq et al., 2017): Proximity Detection, Triangulation and Scene Analysis. The Proximity Detection method is the simplest in terms of implementation, it attributes the target's position to the strongest received signal (Zafari et al., 2017); the Triangulation techniques uses the geometric properties to compute the target location (Jacq et al., 2017), while the Scene Analysis uses scene details computed from a particular point of view to match patterns (Terán et al., 2017). Since the localization system has to guarantee the identification of a patient in an environment, but not its accurate position within it, the selected 
methodology for this work is based on Proximity Detection. The system has been modeled by reversing the traditional paradigm of indoor localization: Bluetooth Beacons are the mobile devices inside the structure, while the location is determined through a system of antennas arranged in both internal and external environments.

The remainder of the paper is organized as follows: Section 2 introduces the requirements of an indoor localization system specially targeted for a residential care, Section 3 overviews the implemented system, while Section 4 concludes with a brief summary and mentions future work.

\section{REQUIREMENTS}

“Il Paese Ritrovato” (Il Paese Ritrovato Project, 2018) is the first village in Italy exclusively designed by the La Meridiana cooperative (La Meridiana, 2018) as a pioneering care facility for people affected by dementia, located in the city of Monza. As a gated model village, "Il Paese Ritrovato" is a selfcontained community where patients are free to move and interact with each other in wide open spaces, and where structures have been specifically designed to meet the patient's needs, freeing them to the burden of feeling slaves of the disease. The residence consists of eight apartments with eight private rooms each. Each apartment also has common rooms such as the kitchen, the dining room and two living areas. A square is located in the center of the village, where residents can socialize visiting special buildings dedicated to them: a bar, a hairdresser, a cinema and a church. La Meridiana has thought a new concept of residence, where everything is designed to support the lives of Alzheimer's patients. Thanks to the collaboration of many research institutions, the structure is constantly looking for innovation with cutting-edge technological systems, environmental and service design.

In order to preserve the independence of the patients without reducing their safety inside the structure, the number of caregivers to assist patients has been redesigned to one every eight, which coincides with one available caregiver for each apartment. Considering the dimension of the structure, the unconventional freedom of the patients and the limited amount of human resources, the activities of the caregivers have to be simplified through the use of a system developed specifically for the supervision of the structure. The main need expressed by the structure is to have a mobile application able to monitor the patients' position, their meals and medicines. Further-

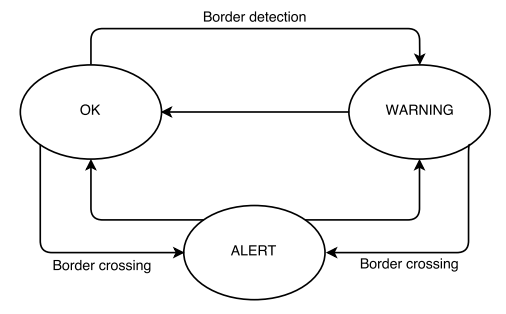

Figure 1: Push Notifications on the caregiver smartphone rose if a patient cross virtual fences.

more, an efficient localization system should keep the caregivers updated about the state of the patients (see Figure 1), allowing automatic generation of warnings/alerts if patients approach/cross borders that are not allowed. It is important to remember that the monitored subjects are affected by a degenerative disease that limits their cognitive abilities: the localization system must not require any interaction by the users and must be as non-invasive as possible.

Moreover, a constant monitoring may be useful to obtain a complete analysis comprising medical data resulting from clinical exams (e.g., electrical activity of the heart, blood pressure, oxygen saturation), medical history and also an extremely accurate description of the activities performed as a daily routine. This type of monitoring is at the basis of behavioral drifts' analysis (Veronese et al., 2018); changes in human behavior, such as changes in the attendance of environments or in the order of execution of daily activities could be automatically recognized by the system and reported to caregivers. A crucial aspect of this analysis is the collection of data that must be carried out in a non-invasive but constant way: a progression of the disease may lie behind a change in the patient's daily routine.

\section{LOCALIZATION SYSTEM}

The main purpose of Assistive Technologies is to ensure the safety and well-being of patients, while trying to lighten the workload of caregivers. The idea behind this concept is to track the older person's position considering routine activities and social interactions with a minimal impact on his/her life.

The proposed system consists of an indoor localization module based on iBeacon technology (IBeacon, 2018), fixed antennas, and a mobile application; the entire system has been designed according to the requirements of structures dedicated to the care of people with dementia and Alzheimer's disease. 


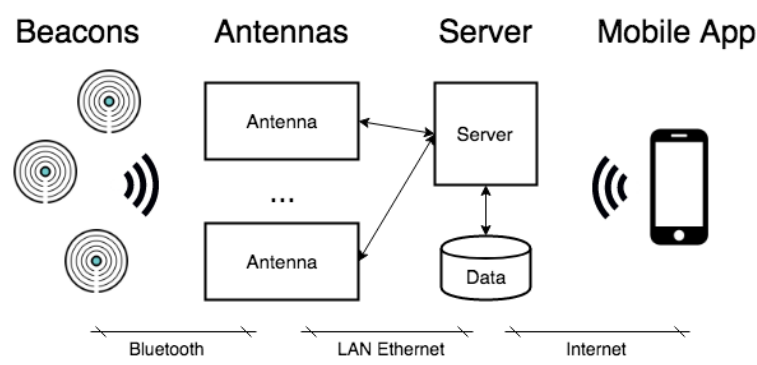

Figure 2: Architecture of the proposed indoor localization system.

\subsection{Architecture}

The proposed localization system is specifically targeted to operate with people affected by mild cognitive disabilities such as dementia. Unfortunately, most of the available localization systems require people to use a device which is characterized by high complexity. The following work moves all the complexity to the system, requiring the users only to wear a disposable bracelet. It is composed by three main components (Figure 2):

- tags: iBeacon devices embedded into plastic bracelets that will be worn every day by the patients of the structure;

- antennas: physical devices that receive the Bluetooth signals emitted by the iBeacons, and transmit the information to the server;

- server: it computes the estimated position of patients given the data received from the antennas.

\subsubsection{Tag}

Each patient of the structure is equipped with a Bluetooth bracelet (iBeacon) and despite aware of being localized while performing daily life activities, he/she does not need to interact with the system.

Beacons are Bluetooth Low Energy (BLE) devices that broadcast packets without the requirement of a paired connection. The payload information meets the standard specified by the iBeacon format and thus contains - among others - a Universal Unique Identifier (UUID), a Major and a Minor values to identify a specific device within a group, and the Broadcasting power; a Beacon can be configured changing its properties, as well as the transmission frequency. Transmitting a packet with a period of 100ms (Apple iBeacon preset), a Beacon has an expected battery life up to 28 weeks; hence, a replace of the batteries every year is necessary.

Antennas are responsible to intercept the beacon messages broadcasted by the tags; the strength of the received packet - Received Signal Strength Indicator
(RSSI) - is subsequently used by the system to estimate the position of tags. However, even if the RSSI value decays exponentially with the distance between transmitter and receiver, this measure is strongly affected by noise. Human body behave as an attenuator of Bluetooth signals, affecting signal strength and lowering accuracy; therefore the position of the beacon with respect to the antennas is fundamental for the preservation of the signal. For this reason, particular attention was paid in the selection of the correct position for the beacon, also considering the invasiveness on the individual. Finally, the choice fell on the use of light-weight 3D-printable plastic bracelets with an insert in which the beacon is positioned.

\subsubsection{Antenna}

The information advertised by iBeacons can be collected only by other BLE enabled mobile devices such as iPhones, Android devices, and also BLE enabled Raspberry Pi, Odroid etc. (Aman et al., 2016). Following the structure needs, the fundamental requirement of the selected device is to have a BLE enable module in order to manage the collection of incoming information from the Beacons, and an Ethernet adapter to connect with the server. Considering the over mentioned requirements, the computational power needed for the operations, and the cost, a Raspberry Pi3 (Raspberry, 2018) has been chosen to act as Antenna: this device is easily available on the market and is certified, ensuring a good reliability. Thus, a Raspberry Pi3 is positioned in the counter top of each patient's room and common spaces, receiving and collecting Bluetooth information broadcasted by the iBeacons.

The antennas continuously scan the Bluetooth Beacons in the environment. Pre-processing is performed on the RSSI data received from each tag, applying a median filter for noise reduction. The filtering window can be set by using a threshold determined during the installation and configuration of the antennas. The resulting data is periodically transmitted to the server via an HTTP request containing the following message:

<idA, UUID, major, minor, power, RSSI>

where $i d A$ corresponds to the antenna's identifier assigned by the server during the initial configuration, UUID, major, minor, and power describe the detected beacon with its filtered RSSI power strength.

The antennas are installed in the ceiling of rooms and corridors, such that the entire surface of the structure is covered. The full coverage of the bluetooth antennas is guaranteed both inside and outside the residence. In order to reduce system wiring, Raspberry 
Pi is alimented through Power over Ethernet (POE) allowing the appliance to be powered using the same cable that connects it to the Ethernet data network. More in details, each apartment is equipped with several Raspberry Pi3 placed in the apartments both in the private rooms, in the corridors and in the common areas such as the kitchen or the living room. Regarding the outdoors, each point of interest such as the cinema, the bar or more simply the open-air areas are mapped with devices.

During the installation of the system, antennas can be configured in room or corridor or external mode. The difference consists in the radius parameter, which determines the sensitivity of the antenna. Increasing the sensitivity of the antennas positioned in the corridor, for example, decreases the probability that the patient is mistakenly positioned in the room if its actual position is in the corridor - as it was required by the residence for security reasons.

\subsubsection{Server}

The server is responsible for receiving messages and correctly saving them to the database. In order to limit the possibility of wall-crossing in the localization (e.g., the detection of a patient at the instant $t$ in his/her room, and at the instant $t+1$ in the dining room, without having been detected him/her in the corridor that connects the two environments), the localization algorithm has been extended to constrain the admissible transitions between adjacent antennas. To meet this need, the architecture has been modeled as a graph whose nodes represent the antennas and the arcs the walkable paths (see Figure 3). Two antennas are therefore said to be adjacent if a user can freely transit from one to the other.

Every second, the server computes the position of all the tags. Considering a generic tag $T A G_{i}$ at time instant $t$, the list of messages $L_{i}(t)$ received from antennas within the last second is retrieved from the database. Those elements with an RSSI value that is lower than a threshold $T_{m}$ in are automatically removed from $L_{i}(t)$. Then, the element with the higher RSSI value, if it exists, is proposed as candidate position $C P_{i}(t)$ to locate the tag. The actual position of the tag $F P_{i}(t)$ is selected by evaluating $C P_{i}(t)$ and $F P_{i}(t-1)$. This evaluation considers the probability of each transition favoring (but not limiting) the transitions between adjacent antennas. Algorithm 1 shows how to compute the position $F P_{i}(t)$ given the candidate position $C P_{i}(t)$ of tag $T A G_{i}$ at time instant $t$, its previous position $F P_{i}(t-1)$ with its likelihood $P\left(F P_{i}(t-1)\right)$. The algorithm requires few threshold values that are pre-initialized but can be tuned from the server web interface:
- $P_{\text {entry}}:$ is the initial likelihood for a new localization;

- $P_{\text {delta }}:$ is the value of likelihood that is added/removed from the total whenever a right/wrong candidate is proposed;

- $P_{\text {min }}$ : is the minimum value of likelihood that is accepted. Under this level, jumping between non adjacent antennas is allowed.

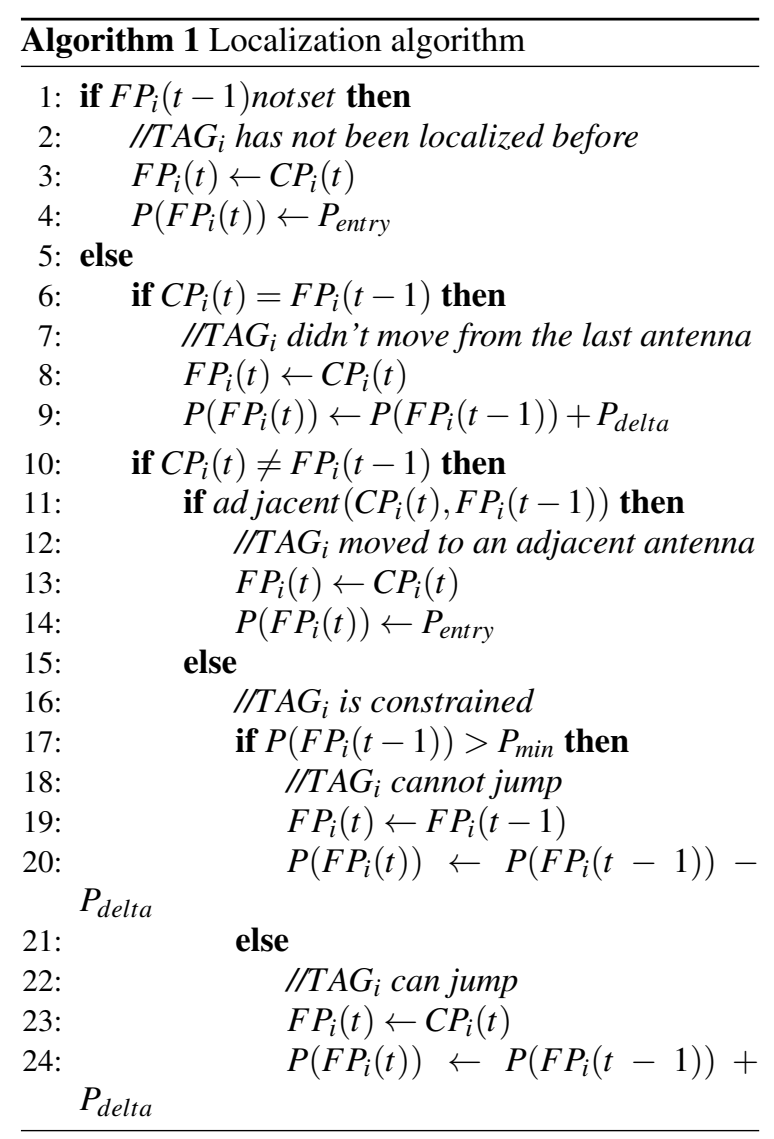

\subsection{Mobile Application}

The mobile application is a primary support for the caregivers. At the beginning of each work shift, the caregiver logs in the application, selects his/her role and immediately sees the position of the patients assigned to him. The main features of the application are listed below:

- Notification: caregivers receive a notification if a patient is approaching or crossing a physical or virtual boundary for the patient (e.g., the exit of the facility, the stairs). With this notification message, the caregiver can assist immediately and, if necessary, can also request the intervention of another colleague in support (see Figure 4). 


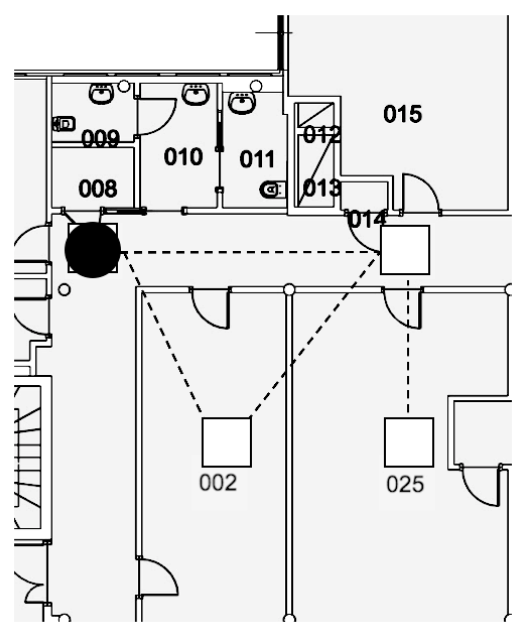

Figure 3: The map is modeled as a graph where nodes (represented as squares) stand for Antennas, and arcs (represented as dashed lines) identify the walkable paths. The black spot indicates the position of a patient that is always localized over a node.


Figure 4: The mobile application raises push notifications to the caregiver whenever a patient violates a virtual fence or another caregiver ask for help.

- Current position: the position of the patients is visible in real time by consulting the "Map" section. The caregiver can, at any time, view the position of the patients assigned to his shift (see Figure 5).



Figure 5: The mobile application allows caregivers to localize the patients in the structure.

- Meals: selecting a patient, the caregiver can visualize and annotate his/her personal data including schedules, preferences, intolerances, etc.

- Medicines: each patient has a personal section linked to medicines with the name of the medicines to be administered, the schedule, and the dosage. The caregiver marks in this section the correct intake of medicines prescribed for the patients.

The Mobile Application has been developed for Android OS using Flutter framework (Google, 2018). Caregivers are equipped with an Android smartphone provided with the Mobile Application already installed.

\subsection{Data}

The gated village "Il Paese Ritrovato" has been inaugurated in February, 2018 and it will host 64 guests by June, 2018. All the guests will be wearing the Bluetooth bracelet to allow their localization, while the caregivers will be provided with a smart card to allow also their localization inside the structure. Moreover, the structure is technologically advanced: smart light bulbs to simulate the sunrise, smart wardrobe to help patients in dressing, smart beds to help people finding the way to toilet during the night, electric shutters and 
several environmental sensors.

Once the village will be operative, it will become a source of data from which to draw for further analysis such as daily habits and interactions, normal and abnormal behaviors, etc.

Attendance of environments Using localization data it is possible to determine how patients relate to the environment; this is particularly important in a village like "Il Paese Ritrovato" that is constantly investing in the research of new strategies to to make patients live better. These data constitute an important source of knowledge to evaluate patients' response to the introduction of new experimental techniques.

Interaction Alzheimer's disease is characterized by difficulties in language, in performing actions, in perception (agnosia), and in the execution of complex movements (apraxia); linguistic problems are mainly characterized by an impoverishment in the vocabulary and a decrease in fluency, leading to a general depletion of oral and written language. Caregivers usually annotate relevant observed behaviors in order to track the progression of the disease and to share these information with their colleague. The use of the data collected in the structure allows continuous monitoring of patient's interaction, providing caregivers with a basis of knowledge on which to base their observations.

Behavior Many researchers are addressing the problem of Activity Recognition and Behavioral Changes leveraging Machine Learning Techniques on data streams; their aim is to provide support in the early diagnosis of chronic diseases and/or anomalies (e.g., falls, strokes). The over mentioned dataset allows the creation of behavioral path for the individual defined as the sequentiality of his/her activities during the day with their duration and location. Alzheimer's is a form of degenerative dementia that becomes progressively disabling for the individual, therefore the timing of the evolution of these events are not known. The creation of this behavioral path allows the identification of a growing discrepancy in the execution of simple activities (e.g., having breakfast) and potentially the delineation a different path of degeneration for each patient. In the intermediate phase of the disease patients slowly are no longer able to perform daily activities, which entail a variation in the daily routine of the patient. The caregiver needs to know any variation in the schedule of activity of the individual, in order to prevent possible abnormal situation.

\section{CONCLUSIONS}

A novel indoor localization system has been designed to answer the needs of residential care for people affected by Alzheimer's disease. The application context is "Il Paese Ritrovato", a health-care facility which is researching for innovative treatments to support the wellbeing of patients. Upon arrival in the structure, patients are equipped with a bracelet containing an iBeacon thanks to which they are monitored during their stay. Data broadcasted by the iBeacons are collected through the use of Raspberry devices acting as receiving antennas and analyzed with a Web Server. The system evaluates the RSSI of the received signal and corrects the computed position with a probabilistic approach to avoid wall-crossing. In the traditional approach, the beacons are positioned permanently while the BLE enabled device is in motion. The system implemented involves a reverse approach: the beacons become the moving devices, to be located thanks to the antennas (BLE enabled devices) arranged in the environments.

The first contribution of the paper is the design of an indoor localization system which is accepted and used on people affected by Alzheimer disease. Thus, caregivers have the possibility to monitor their patients using a mobile application which is able to show their position on the map and notify whenever a patient is violating a virtual fence.

The second contribution of this work is the creation of a new dataset referring to the life of elderly affected by Alzheimer's disease in a controlled environment. This dataset will contain data referring to the localization of people, their interaction with IoT devices, their medicines and meals consumptions, their activities and expert annotations. All the collected data will be extremely important for analysis in the field of Behavioral Drift with the aim of identifying - for example - what triggers an acceleration in the progression of the disease.

This system is currently in an experimental state at the structure built by the La Meridiana cooperative which will open in June 2018.

\section{ACKNOWLEDGEMENTS}

Authors would like to thanks La Meridiana cooperative and Fabrizio Danese who contributed to this work with his Master Thesis in Computer Science at Politecnico di Milano University. 


\section{REFERENCES}

Aman, M. S., Jiang, H., Quint, C., Yelamarthi, K., and Abdelgawad, A. (2016). Reliability evaluation of ibeacon for micro-localization. In 2016 IEEE 7th Annual Ubiquitous Computing, Electronics Mobile Communication Conference (UEMCON), pages 1-5.

Farid, Z., Nordin, R., and Ismail, M. (2013). Recent advances in wireless indoor localization techniques and system. Journal of Computer Networks and Communications, 2013

Google (2018). Flutter framework, https://flutter.io.

IBeacon (2018). Apple informazioni sui servizi di localizzazione, https://support.apple.com/en-gb/HT202880.

Il Paese Ritrovato Project (2018). Cooperativa la meridiana, http://www.cooplameridiana.it/il-paese-ritrovato/.

Jacq, D., Chatonnay, P., Bloch, C., Canalda, P., and Spies, F. (2017). Towards zero-configuration for wi-fi indoor positioning system. In Indoor Positioning and Indoor Navigation (IPIN), 2017 International Conference on, pages 1-8. IEEE.

La Meridiana (2018). Cooperativa Sociale La Meridiana, http://www.cooplameridiana.it.

Raspberry (2018). RaspberryPi 3 - Model B, https://www.raspberrypi.org/products/raspberrypi-3-model-b/.

Tariq, Z. B., Cheema, D. M., Kamran, M. Z., and Naqvi, I. H. (2017). Non-gps positioning systems: A survey. ACM Computing Surveys (CSUR), 50(4):57.

Terán, M., Aranda, J., Carrillo, H., Mendez, D., and Parra, C. (2017). Iot-based system for indoor location using bluetooth low energy. In Communications and Computing (COLCOM), 2017 IEEE Colombian Conference on, pages 1-6. IEEE.

Umphred, D. (2012). Neurological rehabilitation. Elsevier Health, London, 6th edition.

United Nations, D. o. E. and Affairs, S. (2017). World population ageing 2017. Technical report.

Veronese, F., Masciadri, A., Comai, S., Matteucci, M., and Salice, F. (2018). Behavior drift detection based on anomalies identification in home living quantitative indicators. Technologies, 6(1):16.

World Health Organization (2015). Dementia Fact sheet N.362.

Zafari, F., Papapanagiotou, I., Devetsikiotis, M., and Hacker, T. (2017). An ibeacon based proximity and indoor localization system. arXiv preprint arXiv:1703.07876. 